\title{
ENGAGE: Guided Activity-Based Gaming in Neurorehabilitation after Stroke: A Pilot Study
}

\author{
Ann Reinthal, ${ }^{1}$ Kathy Szirony, ${ }^{2}$ Cindy Clark, ${ }^{3}$ Jeffrey Swiers, ${ }^{1}$ \\ Michelle Kellicker, ${ }^{1,3}$ and Susan Linder ${ }^{1,4}$ \\ ${ }^{1}$ School of Health Sciences, Cleveland State University, 2121 Euclid Avenue, Cleveland, OH 44115, USA \\ ${ }^{2}$ Rehabilitation and Sports Therapy, Cleveland Clinic, 9500 Euclid Avenue, Cleveland, OH 44195, USA \\ ${ }^{3}$ Rehabilitation and Sports Therapy, Lakewood Hospital, 14519 Detroit Road, Lakewood, OH 44107, USA \\ ${ }^{4}$ Department of Biomedical Engineering, Cleveland Clinic, 9500 Euclid Avenue, Cleveland, OH 44195, USA \\ Correspondence should be addressed to Ann Reinthal, a.karas@csuohio.edu
}

Received 16 October 2011; Revised 12 January 2012; Accepted 4 February 2012

Academic Editor: Keh-Chung Lin

Copyright () 2012 Ann Reinthal et al. This is an open access article distributed under the Creative Commons Attribution License, which permits unrestricted use, distribution, and reproduction in any medium, provided the original work is properly cited.

\begin{abstract}
Introduction. Stroke is a leading cause of disability in healthy adults. The purpose of this pilot study was to assess the feasibility and outcomes of a novel video gaming repetitive practice paradigm, (ENGAGE) enhanced neurorehabilitation: guided activity-based gaming exercise. Methods. Sixteen individuals at least three months after stroke served as participants. All participants received concurrent outpatient therapy or took part in a stroke exercise class and completed at least 500 minutes of gaming. Primary baseline and posttest outcome measures included the Wolf motor function test (WMFT) and the Fugl-Meyer assessment (FMA). ENGAGE uses a game selection algorithm providing focused, graded activity-based repetitive practice that is highly individualized and directed. The Wilcoxon signed ranks test was used to determine statistical significance. Results. There were improvements in the WMFT $(P=0.003)$ and the FMA $(P=0.002)$ that exceeded established values of minimal clinically important difference. Conclusions. ENGAGE was feasible and an effective adjunct to concurrent therapy after stroke.
\end{abstract}

\section{Introduction}

Stroke is one of the leading causes of disability in the United States with approximately 795,000 people experiencing stroke each year [1]. Many of these individuals are left with residual upper extremity deficits that limit independence with activities of daily living [2] and have been correlated with decreased self-reported quality of life [3]. Current research suggests that task-specific practice results in more functional improvement than traditional neuromuscular rehabilitation (TNR) [4-6]. TNR after stroke has been defined as a typical contemporary practice that includes a combination of active, impairment-based exercises, functional task practice, passive exercise, and activities to enhance sensory inputs $[2,7]$. However, based on an observational study by Lang and colleagues [7], only $25 \%$ of a typical 36-minute upper extremity therapy session involved functional task practice. While the number of practice repetitions required in task-specific practice is not yet fully delineated, it is thought that individuals after stroke must practice for a minimum of several thousand repetitions in order to relearn a task [5].

Several obstacles exist to obtaining adequate practice. First, there are a large number of tasks to be practiced, and while there is some transfer of learning between similar tasks, such as picking up a coffee mug and a can of soda, practicing these tasks would not necessarily be helpful for learning to write with a pencil [5]. Second, repetitive practice of a single task may be boring both to the patient and the clinician [7-10]. Third, the learner must actively engage in task practice; it is not adequate to be passively moved through the motion or activity $[7,11]$. Finally, to ensure optimal movement quality and therapeutic benefit during task practice, a skilled professional must often supervise [8$10]$, making it expensive to provide the necessary hours of training.

Current approaches that provide the opportunity for repetitive practice include constraint-induced therapy (CIT), 
robotics, and virtual reality (VR). In CIT, the individual is forced to practice various tasks repetitively with the hemiparetic arm for six hours a day in a structured environment and for $90 \%$ of waking hours, while the nonhemiplegic arm is constrained. Successful candidates for CIT have higher functioning hands, with some active control of wrist and finger extension $[6,12]$. Both the minimal movement requirement and the intensive structured therapy protocol preclude many patients after stroke from benefiting from this intervention. Robotic instrumentation actively guides movement during repetitive practice, providing partial assistance while also constraining the movement to occur in a specific "correct" movement pattern. These devices have been successfully utilized in people after stroke with both high- and low-level functioning upper extremities primarily in research environments $[13,14]$, but have not been widely adopted in traditional clinical settings. Finally, VR environments provide a three-dimensional computer-generated immersion experience where the "player" completes the task similarly to doing so in the real world. The experience is typically engaging, realistic and has been shown to transfer to a comparable real world activity. VR has been successfully used after stroke and can provide upper extremity repetitive task practice to both lower and higher functioning upper extremities $[15,16]$, but its scalability has been limited due to both size and expense of the equipment requirements.

Video gaming systems such as the Wii and PlayStation II with Eye Toy are commercially available, inexpensive virtual reality-type systems that have been used as an effective adjunct to TNR for individuals with stroke $[8-10,17,18]$. Gaming provides an engaging interaction which is critical in optimizing motor learning [5] and can be easily tailored to individuals with varying degrees of paresis, similarly to some robotic and VR systems, but unlike CIT interventions that are only appropriate for higher functioning arms. Three mechanisms are hypothesized to account for the effectiveness of video gaming in inducing neuroplasticity. First, gaming frequently utilizes more energy than sedentary activities [19] which is important since individuals after stroke are typically less active [20], and enhanced cardiovascular fitness has been shown to improve cortical plasticity $[21,22]$. Second, gaming offers significant bilateral practice opportunities, hypothesized to effectively induce reorganization in contralesional motor networks [23-28]. Finally, during play, the gamer sees an avatar concurrently performing the activity on screen providing visual biofeedback $[18,29]$, similar to techniques using the mirror neuron network after stroke [30, 31]. This network involves the activation of neurons in the parietal and premotor cortex during actual movement and when observing others moving [32].

For this study, we developed a novel method of integrating commercially available video gaming into TNR after stroke called ENGAGE, enhanced neurorehabilitation: guided activity-based gaming exercise. The aims of this study were to (1) examine the feasibility of adding ENGAGE as an adjunct to TNR in a natural clinical practice environment without additional skilled therapy personnel costs using commercially available gaming equipment; (2) examine whether practice completed through ENGAGE gaming leads to improvement in upper extremity function in individuals after stroke; (3) examine effects of covariates on the efficacy of ENGAGE gaming, including concurrent rehabilitation, quantity of gaming time, initial motor and sensory function, and chronicity after stroke; (4) examine whether study participants found gaming to be motivating as compared to home exercise program practice.

\section{Methods}

2.1. Study Design. The study was approved by the Institutional Review Boards for Human Subjects Research at the Cleveland Clinic and Cleveland State University, and informed consent was obtained from each participant. Each participant consented to participating in this study. This study utilized a mixed-methods design, with qualitative measures used to address aim no. 1: feasibility and aim no. 3: covariate analyses. A within-subject pretest posttest design, consistent with the principles of community based participatory research [33] was used to address aim no. 2: improvement in upper extremity function and aim no. 4: motivation. Outcomes were assessed without a control group, evaluating practice in a natural clinical practice environment. Two sites within a regional health system were utilized, representing urban and suburban clinical settings.

2.2. Participants. Patients enrolled in outpatient physical and/or occupational therapy or those participating in a community-based stroke exercise class were eligible to participate. In this pilot study, there was no attempt to control what occurred in concurrent outpatient physical and/or occupational therapy, but activities were consistent with contemporary practice as described by Lang et al. [7] and Miller et al. [2], and included adjuncts such as functional electrical stimulation. Participants in the exercise class completed cardiovascular exercises in addition to basic group stretching and strengthening activities. Inclusion criteria included the following: (1) hemiparesis in the involved upper extremity between Brunnstrom stages 2-6; (2) $\geq$ three months after stroke; (3) sufficient balance to sit safely in unsupported sitting while participating in video gaming; (4) adequate cognitive and communication skills to learn video game within three sessions; (5) the ability to play independently or with support staff assist after set up. Participants were excluded if they had confounding medical interventions during the course of the study, such as upper extremity botox injections. A convenience sample of the 16 participants who completed a minimum of 500 minutes of gaming was included in the outcomes analysis. For this pilot investigation, a minimum of 500 minutes of gaming time was chosen based on the expectation that 12-16 sessions of outpatient rehabilitation is typical before significant improvement occurs, and that each session typically includes a mean of 36 minutes of actual activity [7], resulting in 432-576 minutes of practice. Participant demographics are summarized in Table 1.

2.3. Outcomes. Baseline and posttesting were completed using the upper extremity portion of the Fugl-Meyer 
Table 1: Participant demographics.

\begin{tabular}{lc}
\hline Gender & 8 males; 8 females \\
Side of hemiparesis & 10 right; 6 left \\
Age (years) & $63 \pm 13.9(38-86)$ \\
$\begin{array}{l}\text { Chronicity (months) } \\
\text { Exercise class or } \\
\text { outpatient therapy }\end{array}$ & $26 \pm 25(3-77)\left(5\right.$ subacute; 11 chronic $\left.^{*}\right)$ \\
$\begin{array}{l}\text { Gaming time (minutes) } \\
\text { Concurrent }\end{array}$ & $1089 \pm 619(594-2816)$ \\
occupational therapy & 7 \\
\hline
\end{tabular}

${ }^{*}$ Subacute $3-9$ months after stroke; chronic $>9$ months after stroke.

**All exercise class members chronic.

assessment and the Wolf motor function test as the primary outcome measures in this pilot investigation. Secondary clinical outcome measures included portions of the stroke impact scale and intrinsic motivation inventory. Detailed training and administration manuals were developed and used by all evaluators for the FMA and WMFT. During posttesting, evaluators were blinded to baseline results.

2.3.1. Fugl-Meyer Assessment [34]. The upper extremity motor portion of the Fugl-Meyer assessment (FMA) is a 33item test that assesses reflex integrity and active movement. The FMA is measured on an ordinal scale from 0-2 $(0=$ cannot perform, 1 = performs partially, 2 = performs flawlessly) with a maximum possible score of 66. A study by Lin et al. determined that the FMA has high concurrent validity with $P=0.82-0.96$ [35], high interrater reliability $(\mathrm{ICC}=0.96)$, and high test-retest reliability $(\mathrm{ICC}=0.99)$ [36]. The minimal clinically important difference (MCID) for the upper extremity portion of the FMA is 6.6 points, or $10 \%$ of the total score of 66 [37]. An additional section of the FMA assesses upper extremity sensation, with two items for light touch and 4 items for proprioception. This section uses the same $0-2$ point ordinal scale for a total of 12 points for normal sensation [34].

2.3.2. Wolf Motor Function Test [38]. The timed tasks of the Wolf motor function test (WMFT) include 15 items that assess gross and fine motor tasks. Psychometric properties have been established, with an interrater reliability of $r=$ $0.97(P<0.05)$ and an internal consistency of 92.4\% [38]. Lin and colleagues have determined the MCID to be 1.5 to 2 seconds in subacute stroke [39].

2.3.3. Stroke Impact Scale [40]. The stroke impact scale version 3.0 (SIS) is a self-reported quality of life measure that includes 59 questions over eight domains (strength, hand function, activities of daily living (ADL), mobility, communication, emotion, memory and thinking, and participation). The SIS utilizes a 5-point scale where the person rates the difficulty of each item. For this pilot investigation, the ADL (no. 5) and hand function (no. 7) subsections were chosen as most reflective of change expected as a result of the intervention. Lin and colleagues established MCID levels for the ADL and hand function subscales at 5.9 and 17.8 points, respectively [41].

2.3.4. Intrinsic Motivation Inventory [42]. The interest/ enjoyment subscale of the intrinsic motivation inventory (IMI) is a self-report measure consisting of seven items. Each item is rated on a scale of 1 to 7 according to how true the statement is for the participant. The IMI was used at posttesting, to compare interest/enjoyment in gaming relative to a home exercise program. Validity has been established for this measure [42].

2.4. Intervention. ENGAGE can be distinguished from standard video gaming in the following ways. First, it uses a game selection algorithm that provides focused, carefully graded activity-based repetitive practice of cognitive-perceptualmotor tasks. It offers highly individualized and directed exercise in the form of playing video games in order to specifically address the unique constellation of each individual's neurological impairments after stroke. Second, it uses a limited number of gaming system platforms and games. Third, it is guided by the neuromuscular rehabilitation clinician, yet carried out under the direct supervision of support personnel or, in this study, students, thereby reducing cost while maintaining the intensity and quality of practice. All clinicians who participated as coinvestigators were experienced neurorehabilitation specialists (17-32 years of experience each).

2.4.1. Game Selection Algorithm. A game selection algorithm was developed and used in this pilot study. The first step involved determining the specific and unique needs of each individual after stroke. After completion of an initial examination/evaluation by the clinician, the participant and clinician developed a realistic collaborative functional goal, an important factor in obtaining an engaged commitment to practice $[36,43,44]$. Next, the clinician completed functional task analysis of the goal in conjunction with an assessment of current impairments of body structure/function such as limitations in strength, passive range of motion (PROM), motor control, and sensation that might limit that function/activity. Limiting motor components such as decreased antigravity shoulder flexion were prioritized, as well as critical and interacting nonmotor impairments, such as diminished ability to attend or hemineglect. Games were then selected to bridge the gap between the patient's impairments and the movement required to complete the functional goal. The algorithm allowed for preparatory work on impairments and task components before moving on to practice the actual task, but the gaming itself was always part of a meaningful activity.

In order to efficiently select the appropriate game for a given participant, games utilized in the study were analyzed using a taxonomy representing multiple continua of motor skill difficulty [45] with tables developed to identify these motor components for each game. The first set of factors relating to the environmental demands of playing the game identified whether the environment was stationary (i.e., 
golfing) or in motion (i.e., batting), and if successful play required force modulation and/or accuracy (i.e., archery). The second set of factors involving the body's interaction with the task demands determined which upper extremity joints needed to move in what planes of motion in relation to gravity (i.e., antigravity shoulder flexion with elbow extension during bowling), whether the task needed specific types of grasp and hand/finger manipulation (i.e., drumming versus playing the guitar), if the task itself was continuous (i.e., boxing) or discrete (i.e., golf swing), and whether it required unilateral or bimanual dexterity (i.e., tennis versus canoeing). The ability to complete bimanual task training was another important feature considered in this model, since bimanual function is variable, utilized in more than $54 \%$ of hand activities [46], and has been shown to be an efficacious rehabilitation tool [23, 24, 26-28]. Bimanual skills were further subdivided into four categories: bimanual assisted (i.e., two-handed activity such as golfing with hemiplegic hand ace wrapped to controller); yoked (i.e., baseball batting); reciprocal (i.e., driving); differing (i.e., playing the guitar). In addition to a game's specific upper extremity motor demands, other critical nonmotor factors were considered. For example, cognitive demands were matched with the patient's ability to understand and learn the game. Associated cardiovascular demands were assessed; in general, continuous tasks (i.e., boxing) that require the player to perform a movement without rest had higher demands than games composed of discrete tasks (i.e., bowling) where there is rest between repetitions. Other nonmotor domains included sensation/perception and trunk control/balance. Changes in these nonmotor areas were not specifically monitored outside of the sensory section of the FMA.

\subsubsection{Gaming System Platforms and Games. Therapists'} knowledge of the different game options was essential for appropriate game selection. While an adequate number of games was necessary to meet the varied needs of the different individuals post stroke, this was balanced by the need to know the various game features and requirements. Therefore, games were chosen from six different discs using one of two gaming system platforms, the Sony PlayStation II with EyeToy (games: Play 1, Play 2, and Rock Band) or the Nintendo Wii (games: Wii Sports, Wii Resort, and Wii Play), providing a wide array of options with varying degrees/types of difficulty. The Wii controller contains accelerometers that are actuated by multiplanar tilting, rotation, and acceleration movements, as well as through various control buttons. The EyeToy is a motion-sensitive USB camera that utilizes visual biofeedback by projecting a real time image of the player onto the TV screen. Players see themselves moving within a virtual environment that is superimposed on an image of the actual background [9]. The EyeToy provides more options for individuals with lower functioning arms and/or more cognitive deficits because it is not necessary to activate the accelerometer-based hand controller or manage button controls. Numerous modifications and props were developed to make games more task specific, graded for difficulty, and individualized for the heterogeneous population (i.e.,

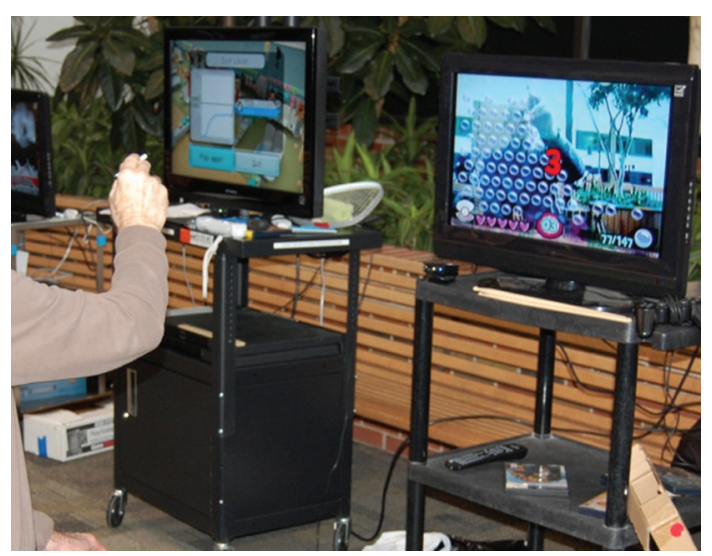

FIgURe 1: Playing Bubblepop on the PlayStation II with EyeToy.

enlarging the wheel during driving, covering unused buttons on the Wii controller). Figure 1 shows an individual playing the game Bubblepop on the PlayStation II with EyeToy, utilizing a precision pinch grasp while holding a pen to pop the bubbles on the screen.

2.4.3. Delegation to Support Personnel. After completing the above analysis, the treating clinician used a goal recording form as a communication tool for the students completing the intervention with all participants. This form listed the collaborative goal, precautions, the primary motor and secondary nonmotor goals, and a list of possible games addressing these goals along with any required modifications. The assisting students were health sciences undergraduate, graduate, and doctor of physical therapy students. The students were required to know how to use each gaming system and play all the games. Training manuals were developed for this purpose and to serve as a quick reference for trouble shooting during gaming sessions. The supervising clinician was always present during gaming, but was treating other patients. Standardized intervention recording logs were used to track what games were played, how much time was spent playing each game, and modifications to the game.

\section{Analysis}

The Wilcoxon signed ranks test was used to calculate differences in the Fugl-Meyer assessment, Wolf motor function test, stroke impact scale, and intrinsic motivation inventory. Data were analyzed using SPSS Version 18 statistical software package. MCIDs were compared to group means for the FMA, WMFT, and SIS (MCID not available for IMI). In addition, for this pilot study, the following covariates were examined: (1) initial level of arm/hand function, as measured by the initial FMA score, (2) time after stroke (subacute versus chronic), (3) concurrent TNR, (4) the amount of gaming time, and (5) sensation.

\section{Results}

4.1. Feasibility. The algorithm developed for game selection based on individualized goals and deficits as presented in 
TABLE 2: Summary of pilot study outcomes.

\begin{tabular}{|c|c|c|c|c|c|c|c|c|}
\hline & $\begin{array}{l}\text { FMA UE } \\
\text { bafore }\end{array}$ & $\begin{array}{l}\text { FMA UE } \\
\text { after }\end{array}$ & $\begin{array}{l}\text { WMFT } \\
\text { before }\end{array}$ & $\begin{array}{l}\text { WMFT } \\
\text { after }\end{array}$ & $\begin{array}{l}\text { SIS no. } 5 \mathrm{ADL} \\
\text { before }\end{array}$ & $\begin{array}{c}\text { SIS no. } 5 \text { ADL } \\
\text { after }\end{array}$ & $\begin{array}{l}\text { SIS no. } 7 \text { hand } \\
\text { before }\end{array}$ & $\begin{array}{l}\text { SIS no. } 7 \text { hand } \\
\text { post }\end{array}$ \\
\hline Mean & $39 / 66$ & $47 / 66$ & $41 \mathrm{sec}$ & $30 \mathrm{sec}$ & $62 \%$ & $64 \%$ & $33 \%$ & $38 \%$ \\
\hline SD & 20 & 20 & 39 & 39 & 20 & 18 & 25 & 25 \\
\hline$P$ value* & & $0.002^{*}$ & & $0.003^{*}$ & & 0.551 & & 0.599 \\
\hline
\end{tabular}

* Statically significant $P<0.05$ with the Wilcoxon signed-ranks test.

Section 2 was feasible. In a given gaming session run by undergraduate and graduate students, the clinician spent an average of 10 minutes giving instructions and assisting with game selection and modification. Overall, the commercially available game options met the needs of a wide range of deficits after stroke and were highly modifiable. However, it was important that the supervising clinician as well as the students was knowledgeable about the different gaming options. About $50 \%$ of the participants needed some hands on assistance from the students during the gaming sessions.

4.2. Improvement in Upper Extremity Function. Table 2 summarizes the means and standard deviations of the outcome measures. The average score on the FMA increased by 8 points $(P=0.002)$, which is greater than the 6.6-point MCID [37]. The mean improvement of 11 seconds on the WMFT $(P=0.003)$ was greater than the 1.5 - to 2 -second MCID in subacute stroke [39]. There was no statistically significant difference in the two subscales of interest in the SIS:ADL $(P=0.551)$ and hand function $(P=0.599)$.

4.3. Covariate Analysis. Several analyses were completed with the primary outcome measures, the FMA and WMFT, to help delineate inclusion criteria for the planned randomized clinical trial (RCT). First, FMA and WMFT results were examined in relation to initial arm function as measured by the FMA scores (Figure 2). As a result of this analysis and in conjunction with research on return of arm function after stroke $[47,48]$, participants were grouped into four groups. Participants who had initial scores of 10 and lower (no. 1 and 2) did not benefit from the intervention, while most individuals in the other three groups $(11-35 ; 36-55 ; 56-66)$ did improve.

In order to determine whether time after stroke affected outcomes, participants were grouped into either subacute (3-9 mo post) or chronic ( $>9$ mo post) categories [3] based on when they began the gaming study. Figure 3 shows that while the subacute group made larger gains, the chronic group also improved.

The effect of concurrent upper extremity traditional neuromuscular rehabilitation was examined. All participants were either receiving concurrent occupational therapy (OT) consisting of traditional upper extremity neuromuscular rehabilitation, outpatient physical therapy which focused primarily on the lower extremity neurorehabilitation, balance, and/or aerobic training, or were participating in an exercise class. As seen in Figure 4, while both groups improved, those individuals receiving concurrent upper extremity traditional neuromuscular rehabilitation had greater improvements.

Since the amount of gaming time was self-selected in this pilot project, its affect on the outcomes was examined. Participants were grouped according to minutes of gaming time as follows: $500-999$ minutes (56\% of participants), $1000-1499$ minutes (25\% of participants), and 1500 minutes or more (19\% of participants). As can be seen in Figure 5, the group who completed the greatest amount of gaming showed the greatest improvement. No significant difference was found between the low and middle gaming time groups.

Finally, Figure 6 illustrates the changes seen in participants presenting with initial sensory dysfunction on the FMA. All but one of the 11 participants showed improvements in sensation at the time of postintervention testing.

4.4. Motivation. Motivation to practice was assessed using the IMI. This was statistically significant $(P=0.001$ using the Wilcoxon signed-ranks test) with the mean increasing from $5.3 / 7(\mathrm{sd}=1.0)$ for the home exercise program to 6.4/7 $(s d=0.7)$ for gaming.

\section{Discussion}

In this pilot study, we found that participants were highly engaged in the gaming activities, resulting in improved participation in this voluntary adjunctive intervention. This, in turn, led to the completion of significant quantities of repetitive goal-directed practice at levels rarely obtained in traditional outpatient upper extremity neurorehabilitation [7]. The virtual reality literature discusses the concept of "presence" as a form of positive, active engagement that occurs during the VR experience [49]. Our observations are consistent with work by Rand and colleagues [9] who noted a high sense of presence during gaming for their participants.

It was feasible to use the ENGAGE protocol to provide large quantities of meaningful and engaging practice in a realistic clinical environment. While not formally measured throughout the study, actual practice repetitions varied from only a few per minute for games like golf and bowling to over 50 repetitions per minute in games such as boxing and drumming. The game selection algorithm allowed for preparatory work on impairments and task components as part of a meaningful activity before progressing to practicing the actual task. This may clarify the recent discussion about the effectiveness of upper extremity task practice [5053], since we hypothesize that an underappreciated critical component is meaningful and engaged practice rather than 


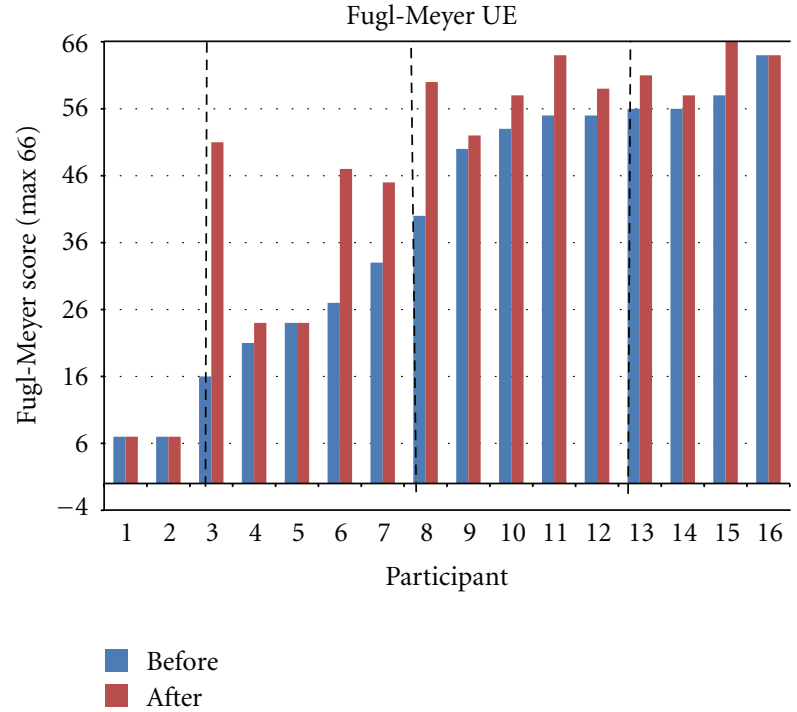

(a)

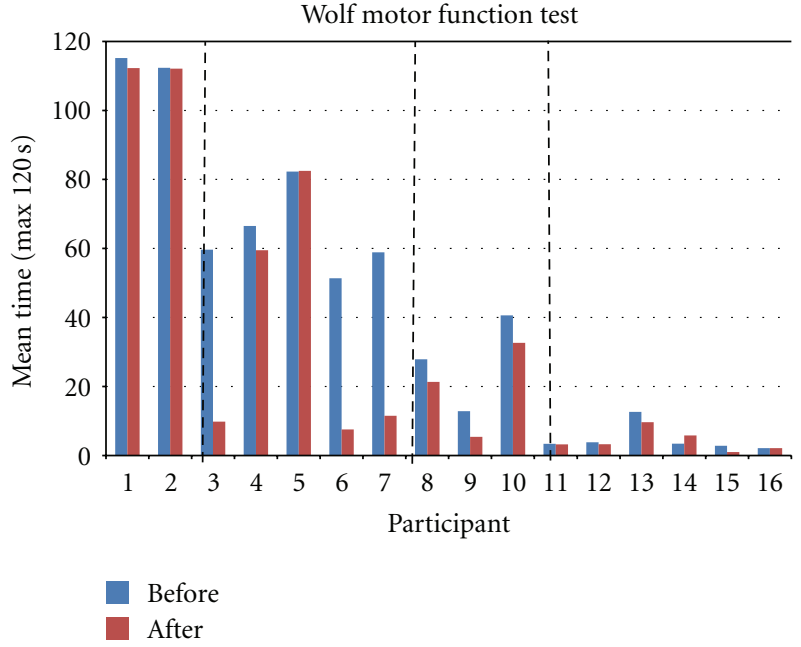

(b)

FIgURE 2: Motor outcomes based on initial UE function on FMA.

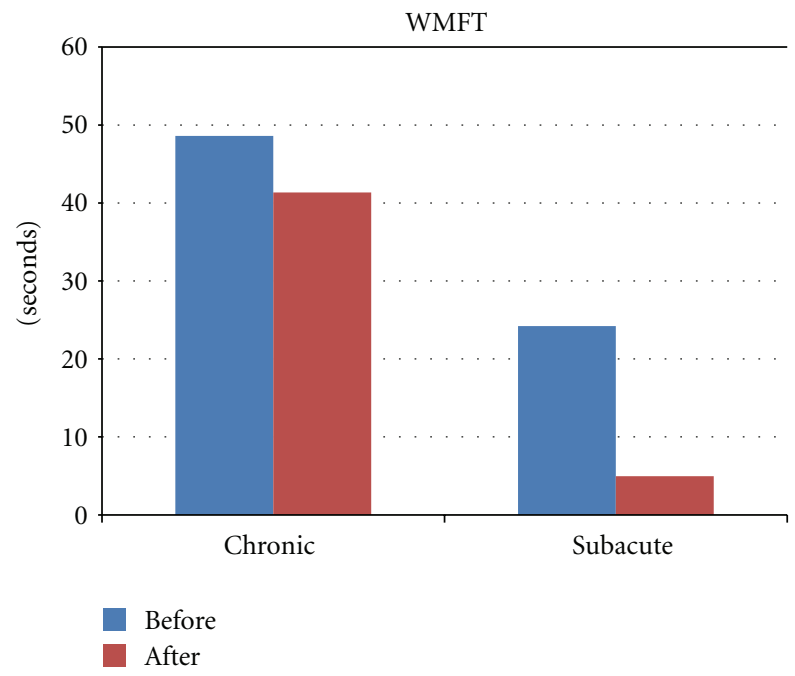

(a)

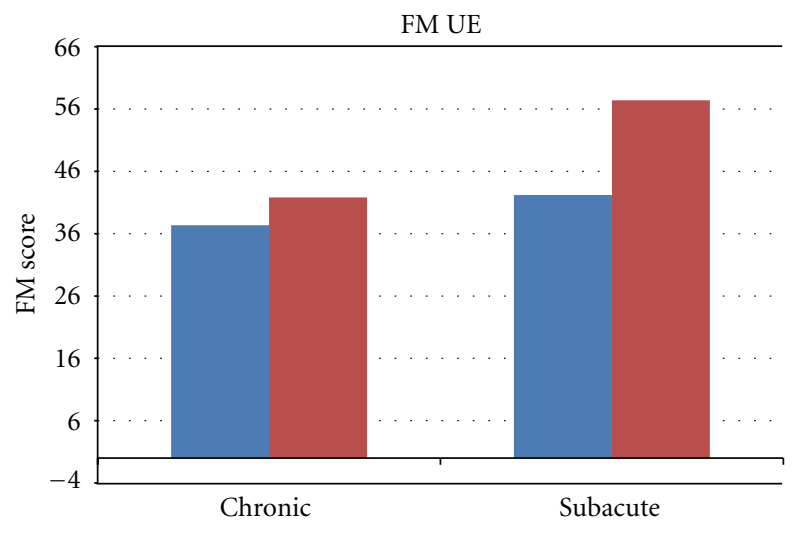

(b)

Figure 3: Effect of time after stroke on motor outcomes.

just task specificity. Of additional importance in this small pilot sample is the fact that this intervention resulted in significant changes in motor function for individuals with lower functioning upper extremities (initial FMA upper extremity scores between 11-35) in addition to those with higher function, including some hand and wrist movement. These lower functioning individuals would not be candidates for CIT [6]. At present, the primary evidence-based approaches for these individuals are robotics and virtual reality interventions which are typically expensive and not universally available to the clinical community $[14,15,54]$. The ENGAGE protocol, however, uses inexpensive, commercially available video gaming equipment in a clinically feasible protocol. In our upcoming randomized clinical trial, we plan to include individuals with upper extremity FMA scores of $>10$ at three months or more after stroke since those with lower motor function did not appear to benefit from the protocol. It is probable that some baseline level of motor function is necessary to actively participate in the gaming intervention. In addition, the single very high functioning individual (initial FMA score of 64/66) did not improve on our motor outcome measures. Therefore, we plan to exclude individuals with FMA scores $>63$ because few of the games require high levels of dexterity practice to sufficiently challenge such an individual.

Coordinating gaming with simultaneous upper extremity neuromuscular rehabilitation improved outcomes in this pilot study. Thus, our upcoming trial using the ENGAGE 


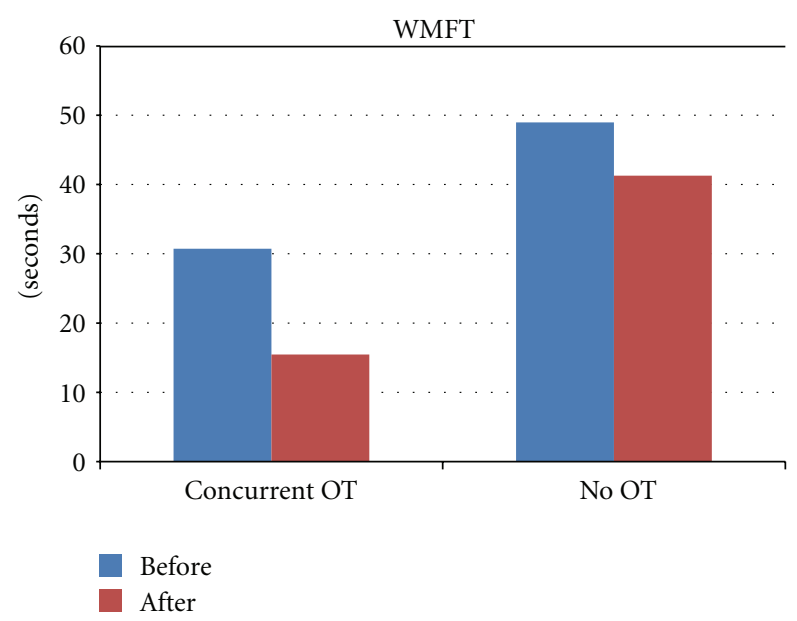

(a)

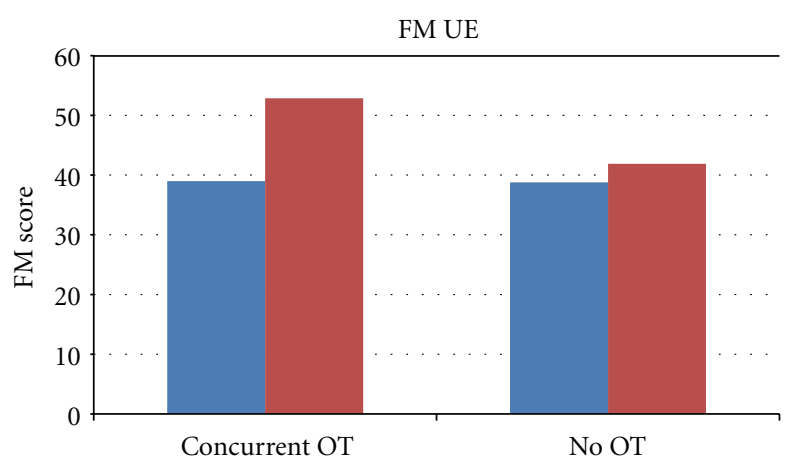

(b)

FIGURE 4: Effect of concurrent upper extremity traditional neuromuscular rehabilitation (OT) on motor outcomes.

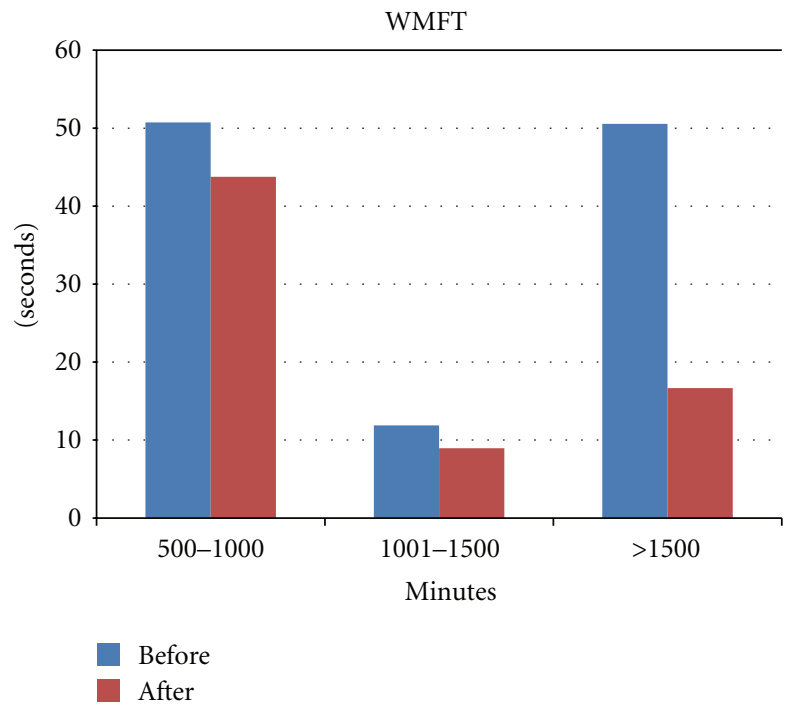

(a)

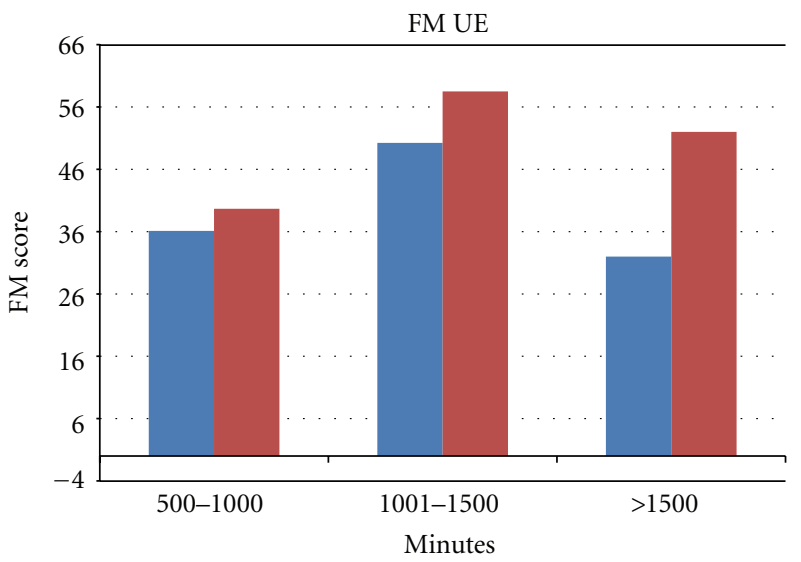

(b)

FIGURE 5: Effect of gaming time on motor outcomes.

protocol will include two intervention arms, each with a different percentage of concurrent upper extremity rehabilitation, to better quantify the dosage of skilled versus adjunctive therapy necessary to result in clinically significant changes in motor function. It offers a novel way to use gaming to substantially increase repetitive practice opportunities at a reduced cost compared to conventional practice [7], while remaining highly individualized and targeted.

The ENGAGE protocol was found to be a cost-effective intervention, due in large part to the ability to utilize students and support staff. In addition to the time required to train clinicians and staff, expense of equipment must be considered. We were able to use only two different gaming system platforms, the Wii and PlayStation II, along with six different game discs on the two systems. This provided a wide selection of gaming options, allowing us to meet the needs of individuals at various levels of arm function, to provide variability of practice opportunities, and to prevent the boredom that often occurs when completing one task repetitively. While it was critical for treating clinicians to be familiar with all game options, limiting game selections meant that busy clinicians could learn the different game alternatives in a reasonable amount of time.

Our data analysis revealed several unexpected results. First, we measured upper extremity sensation using the FMA as a potential covariate. We found that 11 out of the 12 participants with sensory loss improved on this section of the FMA postgaming. While we know that sensory function is a critical component in motor learning [55], there is little consensus in our research literature about interventions 


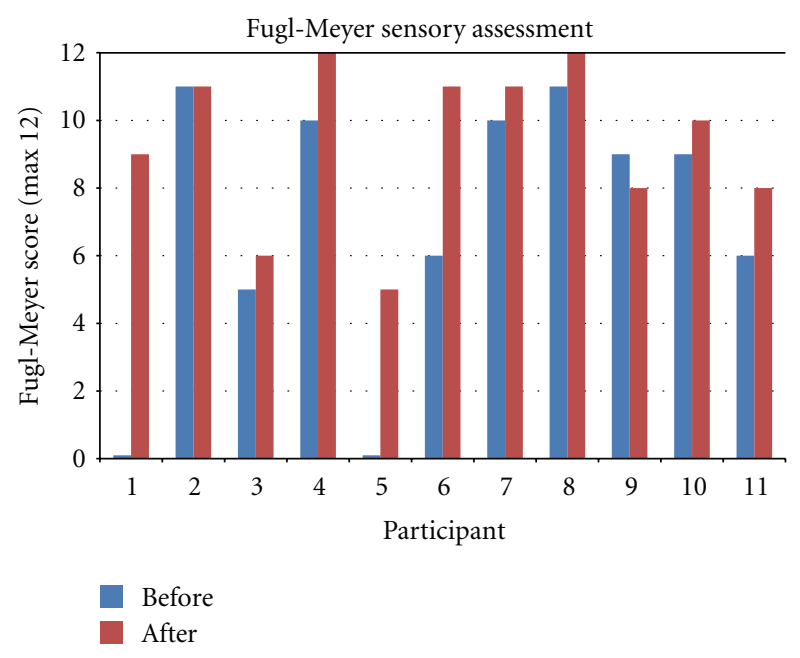

FIGURE 6: Changes in FM sensory assessment for participants presenting with initial dysfunction.

to improve sensation after stroke [56]. These preliminary results warrant more systematic evaluation of sensory function in our upcoming trial. Second, we hypothesized that the subacute group would improve significantly more than the chronic group. Although this pattern did emerge, the difference was small, supporting the theory that many individuals after stroke with upper extremity dysfunction learn not to use the hemiplegic arm and hand [57] and that at present, we do not provide adequate interventions in this area [7]. Based on these pilot results, we will continue to enroll individuals with both subacute and chronic stroke since both groups demonstrated clinically significant levels of change. In this convenience sample representative of the outpatient population of the two participating facilities, half of the outpatient participants were at least six months after stroke. Finally, the one area where participants did not change was the SIS, which is a reflection of the participant's perception of their function, rather than an actual measure of function [40]. Examining the results of only the subacute group, we measured changes beyond the MCID of 5.9 in the ADL subsection (increased from 58.5 to 71), but not beyond the MCID of 17.8 for the hand subsection (increased from 42 to 44) [41]. This can be explained by the fact that several of these individuals did not regain functional use of the hemiplegic hand. Also, it is hypothesized that individuals in the subacute group may have become more aware of the degree of deficit over time after discharge home. The lack of change in the chronic group is potentially a reflection of a more stable perception in their function during the longer time since the stroke event.

This study has a number of limitations. As a pilot clinical outcomes study, there was no control group and no control for concurrent therapy. Additionally, the amount of gaming time was self-selected by each individual participant. These factors will be controlled in our upcoming trial. Dosage questions are unanswered by this study, especially since the 500-999-minute and 1000-1499-minute practice groups made similar gains. The use of gaming time rather than actual practice repetitions may account for this finding, since there is a large variation in repetitions between slowpaced games such as golf and bowling and fast-paced games such as boxing and sword fighting. An accelerometer-based system has been designed as part of this pilot study and will be used in our upcoming trial to quantify the amount of practice required for clinically relevant and functional change [58]. Based on these preliminary data, the protocol for our upcoming trial has been designed to include two intervention arms examining a dosage of one additional hour of gaming for each of twelve weeks, as compared to an hour of TNR. This will provide a total of 720 additional minutes of gaming time or TNR in conjunction with two weekly sessions of TNR. A power analysis has been completed on these preliminary data for this noninferiority hypothesis. A followup testing session one month after completion of the gaming intervention will also be included.

Second, although all raters were trained and used the detailed administration manual developed for the FMA and WMFT, there were multiple evaluators during this study, and interrater reliability was not specifically assessed. The protocol for our upcoming trial includes a blinded evaluator who has been standardized in the administration of the outcome measures to complete all baseline and postintervention testing.

Third, postural stability was not formally assessed in this investigation although improvements were observed that were potentially aided by the gaming intervention. These observations warrant a more systematic evaluation of postural stability in the planned subsequent RCT.

Finally, more objective measures of engagement are needed, as a ceiling effect might have occurred with the IMI. Anecdotally, we, as a group of experienced clinicians, rarely observed a level of engagement in traditional neurologic rehabilitation as witnessed during gaming. We feel the IMI results may have been confounded by the socialization that occurred during gaming but not during home exercise.

\section{Conclusion}

In this pilot investigation, we found that the use of ENGAGE protocol was feasible in a clinical environment. There was a statistically significant improvement in upper extremity function as measured by the upper extremity portion of the FMA and by the WMFT, and participants were motivated to use this gaming protocol. Dosage of gaming time remains unclear, but individuals who completed at least 500 minutes of the intervention demonstrated improvements in function. In future work, the ENGAGE protocol will be used as an adjunct to concurrent upper extremity rehabilitation.

\section{Acknowledgments}

This work was supported by a Faculty Research Development Grant and a Summer Engaged Learning Grant, both from Cleveland State University. In addition, we would like to acknowledge the assistance of multiple undergraduate, graduate, and doctor of physical therapy students from 
Cleveland State University who assisted in the actual gaming work with the participants. Finally, the project could not have been completed without the collaboration, assistance, and support of the clinicians at the Cleveland Clinic Main Campus and Lakewood Hospital.

\section{References}

[1] V. L. Roger, A. S. Go, D. M. Lloyd-Jones et al., "Heart disease and stroke statistics-2011 update: a report from the American Heart Association," Circulation, vol. 123, no. 4, pp. e18-e19, 2011.

[2] E. L. Miller, L. Murray, L. Richards et al., "Comprehensive overview of nursing and interdisciplinary rehabilitation care of the stroke patient: a scientific statement from the American heart association," Stroke, vol. 41, no. 10, pp. 2402-2448, 2010.

[3] D. S. Nichols-Larsen, P. C. Clark, A. Zeringue, A. Greenspan, and S. Blanton, "Factors influencing stroke survivors' quality of life during subacute recovery," Stroke, vol. 36, no. 7, pp. 1480-1484, 2005.

[4] L. Boyd and C. Winstein, "Explicit information interferes with implicit motor learning of both continuous and discrete movement tasks after stroke," Journal of Neurologic Physical Therapy, vol. 30, no. 2, pp. 46-59, 2006.

[5] J. A. Kleim and T. A. Jones, "Principles of experiencedependent neural plasticity: implications for rehabilitation after brain damage," Journal of Speech, Language, and Hearing Research, vol. 51, no. 1, pp. S225-S239, 2008.

[6] S. L. Wolf, C. J. Winstein, J. P. Miller et al., "Effect of constraint-induced movement therapy on upper extremity function 3 to 9 months after stroke: the EXCITE randomized clinical trial," Journal of the American Medical Association, vol. 296, no. 17, pp. 2095-2104, 2006.

[7] C. E. Lang, J. R. MacDonald, D. S. Reisman et al., "Observation of amounts of movement practice provided during stroke rehabilitation," Archives of Physical Medicine and Rehabilitation, vol. 90, no. 10, pp. 1692-1698, 2009.

[8] S. Flynn, P. Palma, and A. Bender, "Feasibility of using the Sony PlayStation 2 gaming platform for an individual poststroke: a case report," Journal of Neurologic Physical Therapy, vol. 31, no. 4, pp. 180-189, 2007.

[9] D. Rand, R. Kizony, and P. T. L. Weiss, "The sony playStation II eye toy: low-cost virtual reality for use in rehabilitation," Journal of Neurologic Physical Therapy, vol. 32, no. 4, pp. 155163, 2008.

[10] G. Yavuzer, A. Senel, M. B. Atay, and H. J. Stam, “'Playstation eyetoy games' improve upper extremity-related motor functioning in subacute stroke: a randomized controlled clinical trial," European Journal of Physical and Rehabilitation Medicine, vol. 44, no. 3, pp. 237-244, 2008.

[11] M. Lotze, C. Braun, N. Birbaumer, S. Anders, and L. G. Cohen, "Motor learning elicited by voluntary drive," Brain, vol. 126, no. 4, pp. 866-872, 2003.

[12] S. L. Wolf, "Revisiting constraint-induced movement therapy: are we too smitten with the mitten? Is all nonuse "learned"? And other quandaries," Physical Therapy, vol. 87, no. 9, pp. 1212-1223, 2007.

[13] R. N. Barker, S. G. Brauer, and R. G. Carson, "Training of reaching in stroke survivors with severe and chronic upper limb paresis using a novel nonrobotic device: a randomized clinical trial," Stroke, vol. 39, no. 6, pp. 1800-1807, 2008.

[14] E. M. Frick and J. L. Alberts, "Combined use of repetitive task practice and an assistive robotic device in a patient with subacute stroke," Physical Therapy, vol. 86, no. 10, pp. 13781386, 2006.

[15] J. H. Crosbie, S. Lennon, M. D. J. McNeill, and S. M. McDonough, "Virtual reality in the rehabilitation of the upper limb after stroke: the user's perspective," Cyberpsychology and Behavior, vol. 9, no. 2, pp. 137-141, 2006.

[16] H. Sveistrup, "Motor rehabilitation using virtual reality," Journal of NeuroEngineering and Rehabilitation, vol. 1, article 10, 2004.

[17] L. Y. Joo, T. S. Yin, D. Xu et al., "A feasibility study using interactive commercial off-the-shelf computer gaming in upper limb rehabilitation in patients after stroke," Journal of Rehabilitation Medicine, vol. 42, no. 5, pp. 437-441, 2010.

[18] G. Saposnik, R. Teasell, M. Mamdani et al., "Effectiveness of virtual reality using wii gaming technology in stroke rehabilitation: a pilot randomized clinical trial and proof of principle," Stroke, vol. 41, no. 7, pp. 1477-1484, 2010.

[19] L. Graves, G. Stratton, N. D. Ridgers, and N. T. Cable, "Energy expenditure in adolescents playing new generation computer games," British Journal of Sports Medicine, vol. 42, no. 7, pp. 592-594, 2008.

[20] D. Rand, J. J. Eng, P. F. Tang, J. S. Jeng, and C. Hung, "How active are people with stroke?: use of accelerometers to assess physical activity," Stroke; a journal of cerebral circulation, vol. 40, no. 1, pp. 163-168, 2009.

[21] S. J. Colcombe, A. F. Kramer, K. I. Erickson et al., "Cardiovascular fitness, cortical plasticity, and aging," Proceedings of the National Academy of Sciences of the United States of America, vol. 101, no. 9, pp. 3316-3321, 2004.

[22] A. F. Kramer and K. I. Erickson, "Capitalizing on cortical plasticity: influence of physical activity on cognition and brain function," Trends in Cognitive Sciences, vol. 11, no. 8, pp. 342$348,2007$.

[23] J. H. Cauraugh, S. A. Coombes, N. Lodha, S. K. Naik, and J. J. Summers, "Upper extremity improvements in chronic stroke: coupled bilateral load training," Restorative Neurology and Neuroscience, vol. 27, no. 1, pp. 17-25, 2009.

[24] F. Coupar, A. Pollock, F. van Wijck, J. Morris, and P. Langhorne, "Simultaneous bilateral training for improving arm function after stroke," Cochrane Database of Systematic Reviews, vol. 4, Article ID CD006432, 2010.

[25] L. Piron, A. Turolla, M. Agostini et al., "Exercises for paretic upper limb after stroke: a combined virtual-reality and telemedicine approach," Journal of Rehabilitation Medicine, vol. 41, no. 12, pp. 1016-1020, 2009.

[26] J. Whitall, S. McCombe Waller, J. D. Sorkin et al., "Bilateral and unilateral arm training improve motor function through differing neuroplastic mechanisms: a single-blinded randomized controlled trial," Neurorehabilitation and Neural Repair, vol. 25, no. 2, pp. 118-129, 2011.

[27] C.-Y. Wu, L.-L. Chuang, K.-C. Lin, H.-C. Chen, and P.K. Tsay, "Randomized trial of distributed constraint-induced therapy versus bilateral arm training for the rehabilitation of upper-limb motor control and function after stroke," Neurorehabilitation and Neural Repair, vol. 25, no. 2, pp. 130139, 2011.

[28] A. R. Luft, S. McCombe-Waller, J. Whitall et al., "Repetitive bilateral arm training and motor cortex activation in chronic stroke: a randomized controlled trial," Journal of the American Medical Association, vol. 292, no. 15, pp. 1853-1861, 2004.

[29] G. Saposnik, M. Mamdani, M. Bayley et al., "Effectiveness of virtual reality exercises in stroke rehabilitation (EVREST): rationale, design, and protocol of a pilot randomized clinical 
trial assessing the wii gaming system," International Journal of Stroke, vol. 5, no. 1, pp. 47-51, 2010.

[30] M. E. Michielsen, R. W. Selles, J. N. Van Der Geest et al., "Motor recovery and cortical reorganization after mirror therapy in chronic stroke patients: a phase II randomized controlled trial," Neurorehabilitation and Neural Repair, vol. 25, no. 3, pp. 223-233, 2011.

[31] D. Ertelt, S. Small, A. Solodkin et al., "Action observation has a positive impact on rehabilitation of motor deficits after stroke," NeuroImage, vol. 36, no. 2, pp. T164-T173, 2007.

[32] K. A. Garrison, C. J. Winstein, and L. Aziz-Zadeh, "The mirror neuron system: a neural substrate for methods in stroke rehabilitation," Neurorehabilitation and Neural Repair, vol. 24, no. 5, pp. 404-412, 2010.

[33] M. Viswanathan et al., Community-Based Participatory Research: Assessing the Evidence. Evidence Report/Technology Assessment No. 99 A.f.H.R.a. Quality, Editor, RTI-University of North Carolina Evidence-based Practice Center, Rockville, Md, USA, 2004.

[34] A. R. Fugl Meyer, L. Jaasko, and I. Leyman, "The post stroke hemiplegic patient. I. A method for evaluation of physical performance," Scandinavian Journal of Rehabilitation Medicine, vol. 7, no. 1, pp. 13-31, 1975.

[35] J. H. Lin, M. J. Hsu, C. F. Sheu et al., "Psychometric comparisons of 4 measures for assessing upper-extremity function in people with stroke," Physical Therapy, vol. 89, no. 8, pp. 840-850, 2009.

[36] K. Shepherd and G. Jensen, Handbook of Teaching for Physical Therapists, Butterworth-Heinemann, Boston, Mass, USA, 2nd edition, 2002.

[37] D. J. Gladstone, C. J. Danells, and S. E. Black, “The Fugl-meyer assessment of motor recovery after stroke: a critical review of its measurement properties," Neurorehabilitation and Neural Repair, vol. 16, no. 3, pp. 232-240, 2002.

[38] S. L. Wolf, P. A. Catlin, M. Ellis, A. L. Archer, B. Morgan, and A. Piacentino, "Assessing Wolf Motor Function Test as outcome measure for research in patients after stroke," Stroke, vol. 32, no. 7, pp. 1635-1639, 2001.

[39] K. C. Lin, Y. W. Hsieh, C. Y. Wu, C. L. Chen, Y. Jang, and J. S. Liu, "Minimal detectable change and clinically important difference of the wolf motor function test in stroke patients," Neurorehabilitation and Neural Repair, vol. 23, no. 5, pp. 429434, 2009.

[40] P. W. Duncan, R. K. Bode, S. M. Lai, and S. Perera, "Rasch analysis of a new stroke-specific outcome scale: the stroke impact scale," Archives of Physical Medicine and Rehabilitation, vol. 84, no. 7, pp. 950-963, 2003.

[41] K. C. Lin, T. Fu, C. Y. Wu et al., "Minimal detectable change and clinically important difference of the stroke impact scale in stroke patients," Neurorehabilitation and Neural Repair, vol. 24, no. 5, pp. 486-492, 2010.

[42] E. McAuley, T. Duncan, and V. V. Tammen, "Psychometric properties of the intrinsic motivation inventory in a competitive sport setting: a confirmatory factor analysis," Research Quarterly for Exercise and Sport, vol. 60, no. 1, pp. 48-58, 1989.

[43] P. Pound, P. Gompertz, and S. Ebrahim, "A patient-centred study of the consequences of stroke," Clinical Rehabilitation, vol. 12, no. 4, pp. 338-347, 1998.

[44] S. Rosewilliam, C. A. Roskell, and A. D. Pandyan, "A systematic review and synthesis of the quantitative and qualitative evidence behind patient-centred goal setting in stroke rehabilitation," Clinical Rehabilitation, vol. 25, no. 6, pp. 501-514, 2011.
[45] A. Gentile, "Skill acquisition: action, movement, and neuromotor processes," in Movement Science: Foundations for Physical Therapy in Rehabilitation, R. Carr and R. Shepherd, Eds., Aspen Publishers, Gaithersburg, Md, USA, 2000.

[46] S. L. Kilbreath and R. C. Heard, "Frequency of hand use in healthy older persons," Australian Journal of Physiotherapy, vol. 51, no. 2, pp. 119-122, 2005.

[47] G. Kwakkel and B. Kollen, "Predicting improvement in the upper paretic limb after stroke: a longitudinal prospective study," Restorative Neurology and Neuroscience, vol. 25, no. 5-6, pp. 453-460, 2007.

[48] R. H. M. Nijland, E. E. H. Van Wegen, B. C. HarmelingVan Der Wel, and G. Kwakkel, "Presence of finger extension and shoulder abduction within 72 hours after stroke predicts functional recovery: early prediction of functional outcome after stroke: The EPOS cohort study," Stroke, vol. 41, no. 4, pp. 745-750, 2010.

[49] G. Riva, F. Mantovani, and A. Gaggioli, "Presence and rehabilitation: toward second-generation virtual reality applications in neuropsychology," Journal of NeuroEngineering and Rehabilitation, vol. 1, article 9, 2004.

[50] R. L. Birkenmeier, E. M. Prager, and C. E. Lang, "Translating animal doses of task-specific training to people with chronic stroke in 1-hour therapy sessions: a proof-of-concept study," Neurorehabilitation and Neural Repair, vol. 24, no. 7, pp. 620635, 2010.

[51] B. French, L. H. Thomas, M. J. Leathley et al., "Repetitive task training for improving functional ability after stroke," Cochrane Database of Systematic Reviews, no. 4, Article ID CD006073, 2007.

[52] B. French, L. Thomas, M. Leathley et al., "Does repetitive task training improve functional activity after stroke? A Cochrane systematic review and meta-analysis," Journal of Rehabilitation Medicine, vol. 42, no. 1, pp. 9-15, 2010.

[53] A. A. A. Timmermans, A. I. F. Spooren, H. Kingma, and H. A. M. Seelen, "Influence of task-oriented training content on skilled arm-hand performance in stroke: a systematic review," Neurorehabilitation and Neural Repair, vol. 24, no. 9, pp. 858$870,2010$.

[54] A. Mirelman, P. Bonato, and J. E. Deutsch, "Effects of training with a robot-virtual reality system compared with a robot alone on the gait of individuals after stroke," Stroke, vol. 40, no. 1, pp. 169-174, 2009.

[55] A. Shumway-Cook and M. H. Woolacott, Motor Control: Translating Research into Clinical Practice, Lippincott Williams \& Wilkins, Philadelphia, Pa, USA, 3rd edition, 2006.

[56] S. Doyle, S. Bennett, S. E. Fasoli, and K. T. McKenna, "Interventions for sensory impairment in the upper limb after stroke," Cochrane Database of Systematic Reviews, vol. 6, Article ID CD006331, 2010.

[57] E. Taub, "Somatosensory deafferentation research with monkeys: implications for rehabilitation medicine," in Behavioral Psychology in Rehabilitation Medicine: Clinical Applications, L. Ince, Ed., pp. 371-401, Williams \& Wilkins, Baltimore, Md, USA, 1980.

[58] S. Sanka et al., "Utilization of a wrist-mounted accelerometer to count movement repetitions," in Proceedings of the 4th International Conference on Communication Systems and Networks (COMSNETS '12), Bangalore, India, January 2012. 


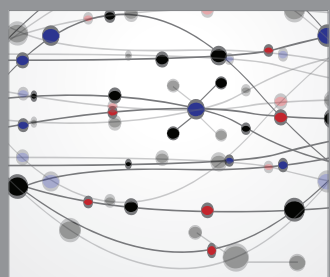

The Scientific World Journal
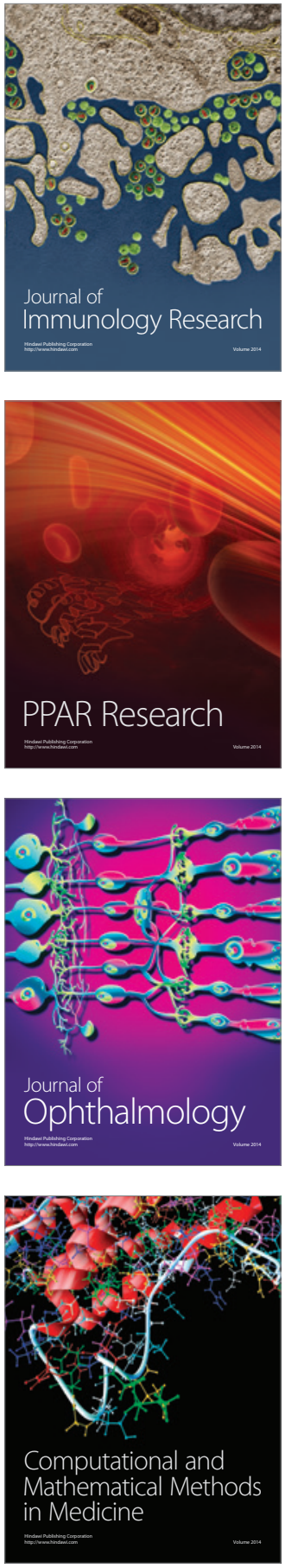

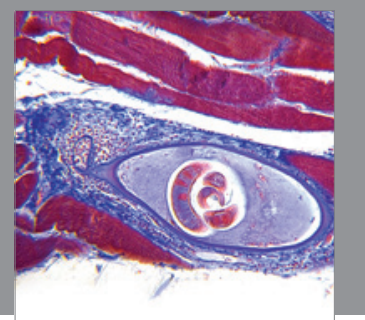

Gastroenterology

Research and Practice
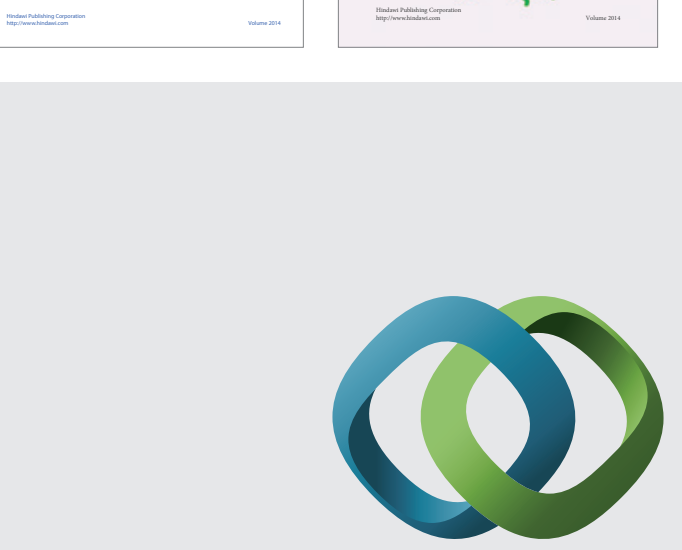

\section{Hindawi}

Submit your manuscripts at

http://www.hindawi.com
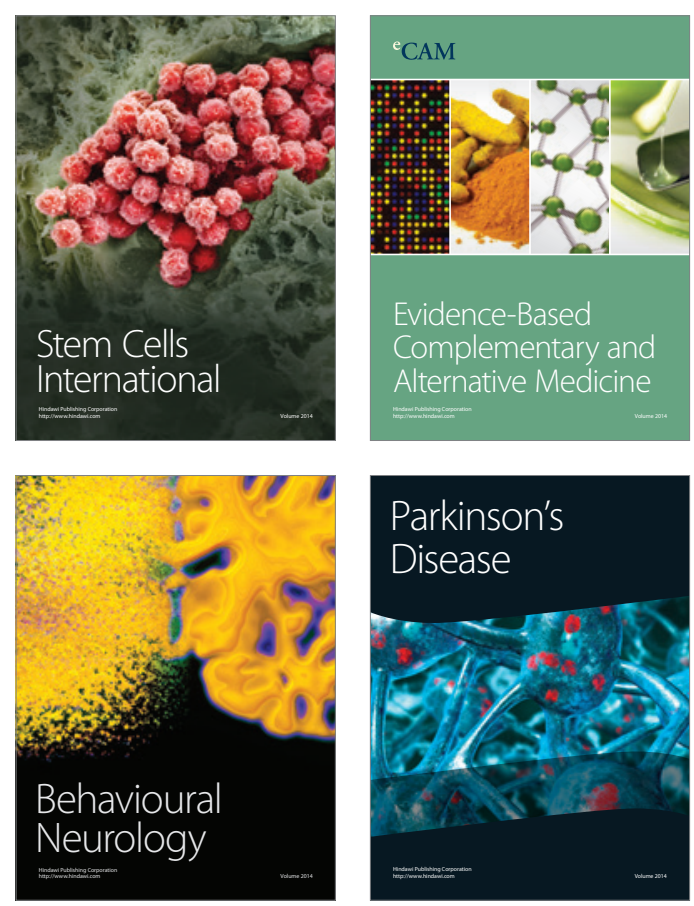

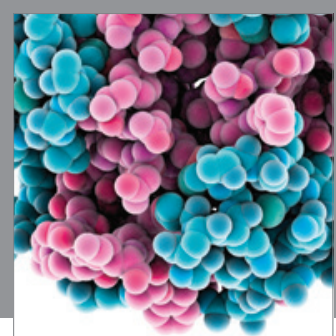

Journal of
Diabetes Research

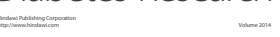

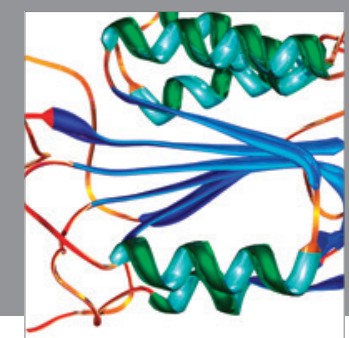

Disease Markers
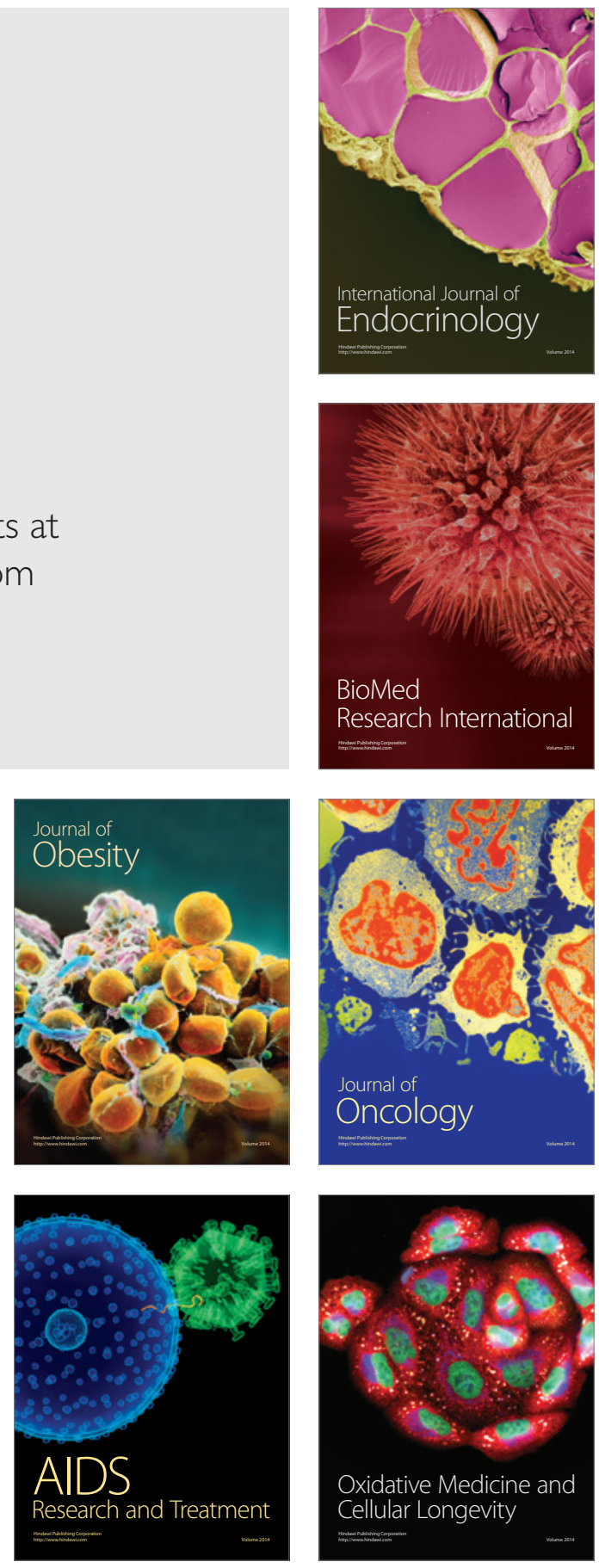